\title{
Enlightening the malaria parasite life cycle: bioluminescent Plasmodium in fundamental and applied research
}

\author{
Giulia Siciliano and Pietro Alano* \\ Dipartimento di Malattie Infettive, Parassitarie ed Immunomediate, Istituto Superiore di Sanità, Rome, Italy
}

The unicellular protozoan parasites of the genus Plasmodium impose on human health worldwide the enormous burden of malaria. The possibility to genetically modify several species of malaria parasites represented a major advance in the possibility to elucidate their biology and is now turning laboratory lines of transgenic Plasmodium into precious weapons to fight malaria. Amongst the various genetically modified

OPEN ACCESS

Edited by:

Luca Cevenini,

University of Bologna, Italy

Reviewed by:

Lucilla lacumin,

University of Udine, Italy Massimo Guardigli,

University of Bologna, Italy

*Correspondence:

Pietro Alano,

Dipartimento di Malattie Infettive,

Parassitarie ed Immunomediate,

Istituto Superiore di Sanità,

Viale Regina Elena n. 299,

00161 Rome, Italy

alano@iss.it

Specialty section:

This article was submitted to

Microbiotechnology, Ecotoxicology

and Bioremediation,

a section of the journal

Frontiers in Microbiology

Received: 20 January 2015

Paper pending published:

23 March 2015

Accepted: 16 April 2015

Published: 11 May 2015

Citation:

Siciliano G and Alano P (2015)

Enlightening the malaria parasite life cycle: bioluminescent Plasmodium

in fundamental and applied research.

Front. Microbiol. 6:391.

doi: 10.3389/fmicb.2015.00391 plasmodia, transgenic parasite lines expressing bioluminescent reporters have been essential to unveil mechanisms of parasite gene expression and to develop in vivo imaging approaches in mouse malaria models. Mainly the human malaria parasite Plasmodium falciparum and the rodent parasite $P$. berghei have been engineered to express bioluminescent reporters in almost all the developmental stages of the parasite along its complex life cycle between the insect and the vertebrate hosts. Plasmodium lines expressing conventional and improved luciferase reporters are now gaining a central role to develop cell based assays in the much needed search of new antimalarial drugs and to open innovative approaches for both fundamental and applied research in malaria.

Keywords: malaria, Plasmodium, bioluminescence, reporter genes, in vivo imaging, cell-based screening assays

\section{Introduction}

Half of the world population is at risk of malaria (World Health Organization, 2013), the most common, and severe parasitic mosquito-borne disease (White et al., 2014). Five species of the protozoan genus Plasmodium infect humans, with Plasmodium falciparum and $P$. vivax causing over 200 million cases/year and $P$. falciparum inflicting virtually all the 6-700,000 annual deaths (2013) recorded mainly in children of Sub-Saharan Africa.

The malaria parasite exhibits a complex life cycle involving an Anopheles mosquito and a vertebrate host (Figure 1). When an infected female mosquito bites a human, the Plasmodium sporozoites travel to the liver and invade hepatocytes, where parasites replicate as hepatic schizonts until several thousand merozoites are produced and released in the bloodstream. In P. vivax, but not in P. falciparum, some liver parasites remain instead quiescent (hypnozoites), resuming replication, and infection after several weeks or months. Upon erythrocyte invasion in the bloodstream Plasmodium parasites undergo asexual replication forming mature schizonts whose rupture releases merozoites that invade new erythrocytes. Some blood stage parasites differentiate instead into male and female gametocytes that, when ingested in the mosquito blood meal, are activated to produce gametes. Gamete fusion in the insect midgut produces a zygote which develops into a motile ookinete, traversing the gut wall, and transforming into an oocyst, where 

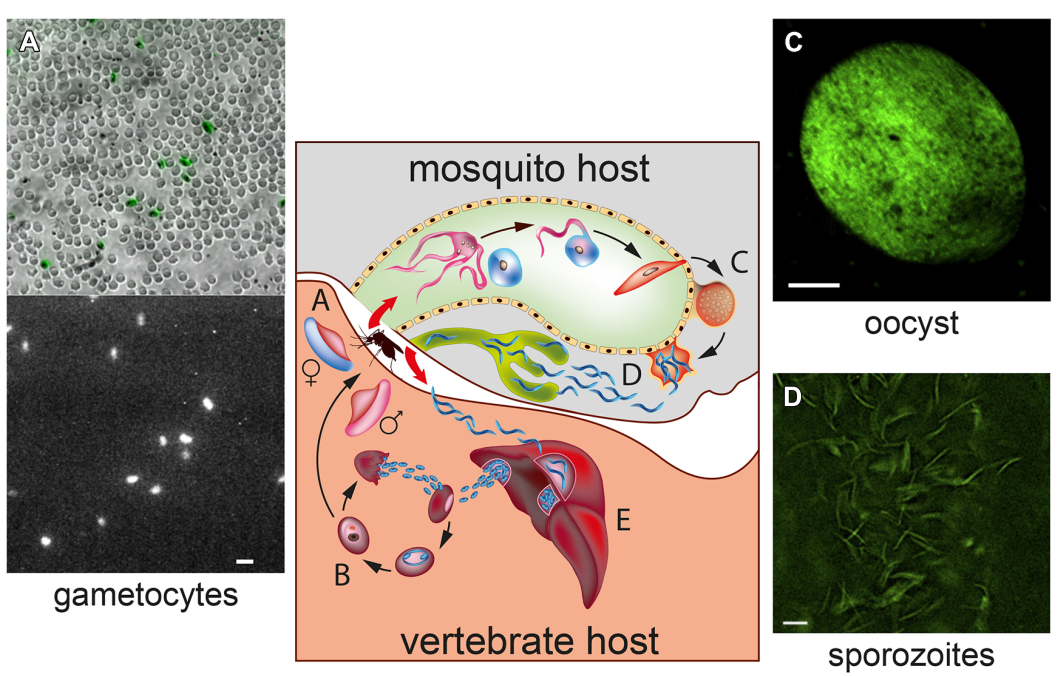

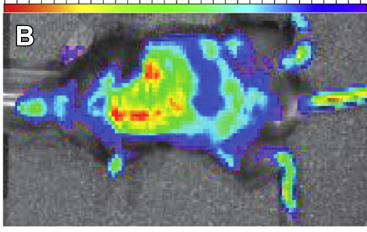

asexual blood stages

FIGURE 1 | Transgenic bioluminescent malaria parasites at differen stages of their development in the vertebrate and mosquito hosts. The central diagram represents the Plasmodium life cycle, showing the progression through the developmental stages of the parasites in the mosquito vector and in the vertebrate host. (A) Bioluminescence imaging (BLI) of individual Plasmodium falciparum gametocytes expressing a click beetle luciferase under a sexual stage-specific promoter. The bright field image shows immobilized gametocytes, highlighted in green, amongst uninfected erythrocytes; the dark field shows the bioluminescence signal of the gametocytes incubated with D-luciferin. Magnification bar: $15 \mu \mathrm{m}$. (Adapted from Cevenini et al., 2014).

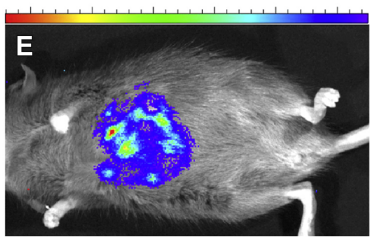

liver stages
(B) BLI of a mouse infected with asexual $P$. berghei parasites expressing a firefly luciferase-green fluorescent protein (GFP) fusion. Heatmap of the bioluminescent signal identifies the sites of accumulation of the parasites (Reproduced with permission from Claser et al., 2011). (C) Fluorescence of a firefly luciferase-GFP fusion protein expressed in $P$. falciparum sporozoites contained in a oocyst and (D) obtained from the dissection of infected mosquito salivary glands. Magnification bar: $5 \mu \mathrm{m}$. (E) In vivo bioluminescent signal obtained by transgenic $P$. falciparum liver stage parasites developing in the chimeric liver of a humanized mouse (C-E) are reproduced with permission from Vaughan et al. (2012). 1000s of sporozoites are produced. The life cycle is closed when sporozoites, migrated from the ruptured oocyst to the mosquito salivary glands, are injected in a new human host by the insect bite.

The pathogenesis of malaria is caused by the asexual blood stages. In the clinical manifestations of $P$. falciparum malaria, the ability of parasites to sequester in the microvasculature of several organs, including the brain, is a major cause of disease severity, and of a fatal outcome (Miller et al., 2002; Milner et al., 2014; Sack et al., 2014). Consequently, the need to cure symptomatic patients traditionally drove efforts toward finding drugs targeting the asexual blood stage parasites, often underestimating the importance of eliminating also the sporozoite, and gametocyte transmission stages or, in $P$. vivax, the hypnozoites. The recent concerning reports from South East Asia of a decreased sensitivity of some $P$. falciparum infections to frontline combination therapies based on artemisinin derivatives is now calling for renewed efforts to address this emergency in the frame of a global strategy to control malaria and eventually eradicate this deadly parasite.
It is possible to cultivate all asexual and sexual blood stages of $P$. falciparum in vitro, unlike P. vivax. Plasmodium species infecting rodents have been also intensely studied as mouse models of aspects of malaria, with $P$. berghei particularly exploited for its amenability to genetic manipulation. In contrast, transgenesis technology has been comparatively more troublesome in $P$. falciparum. This review aims to highlight the importance of Plasmodium transgenic parasites, particularly those engineered with bioluminescent reporters, both in the study of the fundamental biology of Plasmodium and in developing effective antimalarial treatments.

Luciferase enzymes catalyze the light-producing chemical reactions of bioluminescent organisms, in which a luminogenic substrate (e.g., D-luciferin) is oxidized in the presence of ATP, yielding photons. These can be accurately measured by a luminometer with a sensitivity and a virtual absence of background that made bioluminescent reporters potent and versatile tools in biology (Smale, 2010). Luciferases hold a special place in the history of Plasmodium transgenesis: the first plasmid construct to be successfully transfected in 
malaria parasites contained a firefly (Photynus pyralis) luciferase gene whose expression, driven by the promoter of a parasite sexual stage-specific gene, was measured in ookinetes of the bird parasite P. gallinaceum (Goonewardene et al., 1993). Subsequently, luciferase reporters have been used to optimize transfection techniques in Plasmodium parasites (Epp et al., 2008; Hasenkamp et al., 2012), including the introduction of the luciferase from the sea pansy Renilla reniformis, where use of different substrates (D-luciferin and coelenterazine) enabled simultaneous detection of the two parasite produced reporters (Militello and Wirth, 2003; Helm et al., 2010). Since the 1990s, with the stable genetic transformation of different species of Plasmodium (Waters et al., 1997), luciferase reporter genes greatly contributed to elucidate key aspects of malaria infection, from the parasite cellular biology, protein trafficking, gene function, and drug resistance, in several developmental stages throughout the Plasmodium life cycle (Figure 1).

\section{The Plasmodium Life Cycle Marked by Bioluminescent Parasite Developmental Stages}

\section{Plasmodium Mosquito Stages}

Parasite sexual stage development in the mosquito vector is crucial for the transmission of Plasmodium, and elucidating the biology of this process may therefore lead to design novel malaria transmission-blocking strategies. Some studies with bioluminescent parasites highlighted the importance of post-transcriptional regulation acting on stability and translation of several mRNAs, including those encoding major proteins of the gamete and ookinete surface (Mair et al., 2006). Assays with luciferase reporters were for instance fundamental to identify regulatory elements in the transcripts of the P25 and P28 surface proteins of P. gallinaceum and P. falciparum (Golightly et al., 2000; Oguariri et al., 2006).

Plasmodium parasites expressing luciferases also improved tool development for applied studies. A powerful bioassay to determine parasite ability to infect mosquitoes is based on feeding cultured Plasmodium gametocytes to mosquitoes, and it is used to measure effect of transmission blocking drugs or antibodies. This assay is, however, technically demanding and time consuming as the resulting oocysts need to be individually counted in dissected insects. After improvements by using $P$. berghei parasites expressing a green fluorescent protein (GFP) in mosquito stages (Delves and Sinden, 2010), a transgenic line of the human parasite $P$. falciparum line expressing the firefly luciferase in oocysts was developed. In the resulting luminescence-based standard membrane feeding assay (SMFA) the mean luminescence intensity of individual and pooled mosquitoes accurately quantified mean oocyst intensity, eliminating the need for mosquito dissection, and putting the basis for significant SMFA scalability (Stone et al., 2014).

Toward the end of parasite development in the mosquito, the sporozoites produced in the oocyst migrate to the insect salivary glands. Number of salivary gland sporozoites, the only mosquito stages infectious to a mammalian host, is an important index of Plasmodium mosquito development. The construction of a $P$. berghei line where a GFP-luciferase fusion is specifically expressed in sporozoites enabled establishment of a simple and fast assay of sporozoite loads from whole mosquitoes (Ramakrishnan et al., 2012).

\section{Transmission from Mosquitoes: Sporozoites and Liver Stages}

Plasmodium sporozoites injected from an infected mosquito to a human or rodent host start their intracellular development into the liver hepatocytes. This clinically silent stage is the target for prophylactic or vaccine strategies, particularly against $P$. vivax long lasting hypnozoites.

Plasmodium liver stage development has been poorly explored compared to that of blood stages partly because the in vivo and in vitro analyses, respectively, in mouse models and in cultured liver cells, are constrained by the necessity to sacrifice high numbers of mice or by inefficiency of sporozoite infection of cultured liver cells. Transgenic luciferase-expressing sporozoites improved detection strategies introducing bioluminescence imaging (BLI) and in vivo imaging system (IVIS) in the analysis of parasite liver stage development in live mice and in cultured hepatocytes. Real-time BLI requires injection of the luciferin substrate in the mouse or in the dissected organ and an intensified chargecoupled photon counting video camera to measure photon emission (Franke-Fayard et al., 2006; Braks et al., 2013). BLI and IVIS using firefly or sea pansy luciferases have been used for real-time, live monitoring of the progression of rodent parasitic infection in the whole animal or in specific organs (Ploemen et al., 2009; Annoura et al., 2013; Manzoni et al., 2014) and to test activity of drugs targeting liver stage infection, using $P$. yoelii and $P$. berghei transgenic sporozoites in human liver HepG2 or Huh-7 cells and in whole mice (Mwakingwe et al., 2009; Ramalhete et al., 2011, 2014; Derbyshire et al., 2012; Lacrue et al., 2013; Li et al., 2014; Marcsisin et al., 2014; Zuzarte-Luis et al., 2014). To improve these approaches, identification of parasite promoters specifically activated in liver development was achieved in $P$. berghei, also in this case relying on use of transgenic luciferase-promoter fusions (Helm et al., 2010).

The ability to reliably quantify parasite infection in hepatocytes is essential in the development of malaria vaccines. To overcome limitations of qRT-PCR-based quantification, $P$. berghei parasites expressing a GFP-luciferase fusion were introduced to evaluate antimalarial immunity both in vivo, in mice where this was induced by sporozoites unable to proliferate after irradiation or chloroquine prophylaxis, and in vitro in Huh-7 human liver hepatoma cells (Ploemen et al., 2011; Miller et al., 2013). Luciferase expressing P. berghei and P. falciparum sporozoites were also used to assess adequacy of sporozoite attenuation, obtained this time by genetic mutation, respectively, in in vivo murine malaria model and in primary human hepatocytes (Annoura et al., 2012; van Schaijk et al., 2014). These studies highlighted the role of cell mediated immunity mounting against the multiplication-deficient sporozoites. A role for antibody mediated immunity was instead shown by BLI of luciferase-expressing 
sporozoites of the human parasite $P$. falciparum in mice with a humanized liver, showing that infection in this organ was reduced by passive transfer of a monoclonal antibody targeting the sporozoite surface protein CSP (Sack et al., 2014). Finally, P. berghei and $P$. yoelii luciferase transgenic parasites were instrumental to evaluate modes of sporozoite administration, a critical bottleneck in immunization, and challenge protocols (Ploemen et al., 2013).

\section{From the Liver to the Blood: the Asexual Erythrocytic Stages}

Maturation of the liver schizont releases 1000s of merozoites that invade blood stream erythrocytes and starts the asexual, symptomatic blood stage infection. In $P$. falciparum the blood stage schizonts disappear from circulation as they adhere to host ligands on endothelial cells of the microvasculature in several organs, especially in the brain and in the placenta, through parasite proteins expressed on the infected erythrocyte surface, leading to severe pathogenesis such as cerebral malaria or adverse effects during pregnancy. As parasites are observed to accumulate in several organs, including the brain, also in the mouse malaria model, real-time BLI in whole mice or in dissected organs were conducted with $P$. berghei transgenic lines expressing luciferase under a constitutive or a schizont-specific promoter to identify the involved components of the immune system (Franke-Fayard et al., 2005; Amante et al., 2007; Spaccapelo et al., 2010; Claser et al., 2011; Pasini et al., 2013; Imai et al., 2014).

The need to elucidate the mechanisms of malaria pathogenesis directed research on the fundamental biology of parasite asexual development, one important aspect being how the parasite regulates its gene expression. The extremely high $\mathrm{A}+\mathrm{T}$ content of the Plasmodium genomes however, prevented homology based identification of promoters, regulatory elements, and parasite transcription factors, whereas luciferase reporters proved to be of paramount importance in functionally identifying gene promoters and regulatory regions (Horrocks and Kilbey, 1996; Porter, 2002; Militello et al., 2004; Hasenkamp et al., 2013a). This work identified sequences functioning as bi-directional promoters, like the intergenic region of the $P$. berghei elongation factor- $1 \alpha$ $(e f-1 \alpha)$ gene (de Koning-Ward et al., 1999; Fernandez-Becerra et al., 2003) or the intron of the P. falciparum var genes (Epp et al., 2008), or evaluated whether specific promoters from one Plasmodium species were able (Fernandez-Becerra et al., 2003; Ozwara et al., 2003), or unable (Azevedo and del Portillo, 2007) to recruit the transcriptional machinery of a different malaria species. Importantly, luciferase expressing parasites were used to identify regulatory regions governing the expression of the $P$. falciparum polymorphic var genes encoding the parasite sequestration ligands, whose expression switch is responsible for parasite antigenic variation, and immune evasion (Deitsch et al., 1999; Calderwood et al., 2003; Frank et al., 2006; Muhle et al., 2009). In summary, luciferase reporters not only contributed to identify functional elements involved in parasite gene regulation (Bischoff et al., 2000; Militello et al., 2004; López-Estraño et al., 2007; Gopalakrishnan and López-Estraño, 2010; Zhang et al., 2011; Patakottu et al., 2012), but also were essential to select specific promoters in the development of Plasmodium inducible expression systems (de Azevedo et al., 2012; Kolevzon et al., 2014) and to test new regulatory regions in chromosomally integrated luciferase cassettes (Ekland et al., 2011; Weiwer et al., 2011; Che et al., 2012; Khan et al., 2012; Hasenkamp et al., 2013b).

A major effort in the fight against malaria, particularly $P$. falciparum, has been the screening for new antimalarial drugs, an endeavor that the appearance of artemisinin resistance in South East Asia makes dramatically urgent. In the past decades, in vitro methods measuring the incorporation of $\left[{ }^{3} \mathrm{H}\right]$-labeled hypoxanthine and ethanolamine or the activity of parasite Lactate Dehydrogenase have been the standard for $P$. falciparum cell based assays and used in large drug screenings (Fidock, 2010). The demand for high-throughput, nonradioactive assays prompted to exploit also in Plasmodium the high sensitivity and virtual absence of background of luciferase reporters, until recently used in this field only to study expression of the $P$. falciparum multidrug resistance gene $p f m d r 1$ in drug treated parasites (Myrick et al., 2003; Waller et al., 2003). To this aim a $P$. falciparum line expressing the firefly luciferase under the heat shock protein 86 ( $p f h s p 86$ ) gene promoter in asexual stages enabled establishment of a cell-based luciferase drug screening assay in 96w plates (Cui et al., 2008), subsequently adapted to $384 \mathrm{w}$ plate using $10^{5}-10^{6}$ parasites per well (Lucumi et al., 2010). Also P. berghei parasites expressing a firefly luciferase-GFP fusion were used for in vitro and in vivo bioluminescence drug assay, enabling use of animal models to test new drugs in vivo (Franke-Fayard et al., 2008; Lin et al., 2013).

\section{Preparing Departure from the Blood: the Gametocytes}

Plasmodium gametocytes are the parasite sexual stages responsible for the transmission from the vertebrate host to the mosquito. Male and female gametocytes are formed in the bloodstream and, in P. falciparum, they mature in 10 days through five developmental stages. Upon ingestion in the mosquito gut, mature gametocytes promptly differentiate into gametes and fertilization ensures parasite infection in the insect vector. A key priority in the present goal to globally eliminate malaria is to identify new drugs targeting in the bloodstream both the asexual and the sexual stages of the parasite. However, the nonreplicative nature of gametocytes imposed to develop specific cell based screening assays, different from those used for asexual stages. One problem is for instance that of false negative signals due to the persistence of fluorescent reporter or of parasite enzyme activities in unhealthy or dying gametocytes. $P$. falciparum lines expressing a GFP-firefly luciferase gene under gametocyte specific promoters were established (Adjalley et al., 2011) and used in high-throughput screening assays of compounds with anti-asexual stage activity (Lucantoni et al., 2013). Recently, luciferase-based gametocyte assays have been improved by replacing the commonly used commercial luciferase substrates with an ATP-free, non-lysing D-luciferin formulation, yielding assay readouts that more reliably monitored viability and sensitivity to compounds of the treated gametocytes (Cevenini et al., 2014). In this work, absence of parasite cell lysis and the introduction in P. falciparum of the use of a potent luciferase from Pyrophorus plagiophthalamus under a 
gametocyte promoter enabled to perform for the first time BLI at the level of single parasite cells, individually distinguishing live and dead P. falciparum gametocytes (Cevenini et al., 2014).

\section{Multiplexing, Subcellular Localization, Imaging: the Future in the Use of Bioluminescent Malaria Parasites}

Virtually all stages of the complex life cycle of malaria parasites have been enlightened by the use in several studies of luciferase reporters. These engineered parasites provided key answers to fundamental biological questions and now represent important tools for drug screening. Novel potent reporters have already expanded the luciferase repertoire used in P. falciparum beyond the $P$. pyralis and Renilla enzymes (Azevedo et al., 2014; Cevenini et al., 2014), increasing sensitivity and enabling to further reduce parasite numbers in high-throughput screening assays, a non-trivial improvement when using specific stages (e.g., the gametocytes) whose cultivation is technically demanding. Nevertheless exploitation of the full potential of bioluminescent reporters in malaria research is just moving the first steps.

The possibility to tune luciferase emission properties, such as emission wavelength, kinetics or termo- and $\mathrm{pH}$-stability, via random or site-directed mutagenesis or use of enzyme natural variants, led to introduce multicolor bioluminescence in antimalarial drug screening. A green and a red light emitting luciferase from $P$. plagiophthalamus were expressed in $P$. falciparum immature and mature gametocytes, providing for the first time the possibility to simultaneously measure differential, stage specific effects of drugs in a dual-color luciferase assay, and opening the possibility to apply multicolor bioluminescence to any parasite stage in fundamental and applied studies. A dual expression system with distinct luciferases would for instance be valuable in cell based high-throughput screenings to readily identify and discard compounds active against the reporter rather than the target cell (Thorne et al., 2012), as they will most likely affect only one luciferase type.

Another promising application of luciferase reporters is through their fusion to specific signals used by the parasite to traffic proteins in different extra cellular compartments of the infected erythrocyte. As protein export is uniquely regulated in the parasite and is essential for its survival, use of such fusions may be invaluable to screen for compounds targeting this process. Preliminary studies were conducted with the $P$. pyralis enzyme (Burghaus and Lingelbach, 2001) and more recent work established $P$. falciparum lines which express a brighter deep-sea

\section{References}

Adjalley, S. H., Johnston, G. L., Li, T., Eastman, R. T., Ekland, E. H., Eappen, A. G., et al. (2011). Quantitative assessment of Plasmodium falciparum sexual development reveals potent transmission-blocking activity by methylene blue. Proc. Natl. Acad. Sci. U.S.A. 108, E1214-E1223. doi: 10.1073/pnas. 1112037108 shrimp luciferase equipped with sequences driving the reporter in the parasite cytoplasm or in erythrocyte compartments (Azevedo et al., 2014).

In another field of application, the achievement of single parasite cell BLI and the availability of luciferases whose red-shifted light emission is more efficiently detectable from blood and tissues are paving the road to significant progress in analyses of the host-parasite interplay. Co-cultures of different $P$. falciparum stages and human cell types in vitro can provide new insights of the physiology of asexual and sexual stage parasite sequestration. The increased sensitivity achieved in in vivo mouse imaging with a red-shifted luciferase expressed by the unicellular protozoan parasite Trypanosoma brucei (Van Reet et al., 2014) is promising in view of use also in Plasmodium infected mice. Importantly, the increasing availability of humanized mouse models for $P$. falciparum and $P$. vivax infections, supporting development of asexual, and sexual blood stages and of liver stages (Kaushansky et al., 2014) and the use of P. falciparum transgenic lines with a luciferase expressed constitutively (Vaughan et al., 2012) or under stage-specific promoters are expected to answer many unsolved questions.

The wealth of biological information provided by the use of engineered bioluminescent malaria parasites, not to mention those not reviewed here expressing a variety of fluorescent reporters, has been and will most likely continue to be enormous. The confined use of these whole cell biosensors in laboratory settings does not pose regulatory concerns on environmental release. From their aseptic sites of utilization, these genetically modified parasites will nevertheless have the most significant impact in the real world, contrasting the unbearable burden of a worldwide devastating disease.

\section{Author Contribution}

GS drafted and PA edited the manuscript.

\section{Acknowledgments}

Authors are indebted to Drs Luca Cevenini, Elisa Michelini, and Aldo Roda, University of Bologna, Bologna, Italy and Dr. Bruce B. Branchini, Connecticut College, New London, CT, USA, for several insightful discussions along our collaborations, and thank Mr. Cosimo M. Curianò for the figure artwork. Research in the authors' laboratory is supported from grants from the Bill \& Melinda Gates Foundation and the EU EVIMalaR Network of Excellence.

Amante, F. H., Stanley, A. C., Randall, L. M., Zhou, Y., Haque, A., McSweeney, K., et al. (2007). A role for natural regulatory $\mathrm{T}$ cells in the pathogenesis of experimental cerebral malaria. Am. J. Pathol. 171, 548-559. doi: 10.2353/ajpath.2007. 061033

Annoura, T., Chevalley, S., Janse, C. J., Franke-Fayard, B., and Khan, S. M. (2013). Quantitative analysis of Plasmodium berghei liver stages by bioluminescence imaging. Methods Mol. Biol. 923, 429-443. doi: 10.1007/978-1-62703-026-7_30 
Annoura, T., Ploemen, I. H., van Schaijk, B. C., Sajid, M., Vos, M. W., van Gemert, G. J., et al. (2012). Assessing the adequacy of attenuation of genetically modified malaria parasite vaccine candidates. Vaccine. 30, 2662-2670. doi: 10.1016/j.vaccine.2012.02.010

Azevedo, M. F., and del Portillo, H. A. (2007). Promoter regions of Plasmodium vivax are poorly or not recognized by Plasmodium falciparum. Malar. J. 6, 20. doi: $10.1186 / 1475-2875-6-20$

Azevedo, M. F., Nie, C. Q., Elsworth, B., Charnaud, S. C., Sanders, P. R., Crabb, B. S., et al. (2014). Plasmodium falciparum transfected with ultra bright NanoLuc luciferase offers high sensitivity detection for the screening of growth and cellular trafficking inhibitors. PLoS ONE 9:e112571. doi: 10.1371/journal.pone.0112571

Bischoff, E., Guillotte, M., Mercereau-Puijalon, O., and Bonnefoy, S. (2000). A member of the Plasmodium falciparum Pf60 multigene family codes for a nuclear protein expressed by read through of an internal stop codon. Mol. Microbiol. 35, 1005-1016. doi: 10.1046/j.1365-2958.2000.01788.x

Braks, J., Aime, E., Spaccapelo, R., Klop, O., Janse, C. J., and Franke-Fayard, B. (2013). Bioluminescence imaging of $P$. berghei schizont sequestration in rodents. Methods Mol. Biol. 923, 353-368. doi: 10.1007/978-1-62703-026-7_25

Burghaus, P. A., and Lingelbach, K. (2001). Luciferase, when fused to an N-terminal signal peptide, is secreted from transfected Plasmodium falciparum and transported to the cytosol of infected erythrocytes. J. Biol. Chem. 276, 26838-26845. doi: 10.1074/jbc.M100111200

Calderwood, M. S., Gannoun-Zaki, L., Wellems, T. E., and Deitsch, K. W. (2003). Plasmodium falciparum var genes are regulated by two regions with separate promoters, one upstream of the coding region and a second within the intron. J. Biol. Chem. 278, 34125-34132. doi: 10.1074/jbc.M213065200

Cevenini, L., Camarda, G., Michelini, E., Siciliano, G., Calabretta, M. M., Bona, R., et al. (2014). Multicolor bioluminescence boosts malaria research: quantitative dual-color assay and single-cell imaging in Plasmodium falciparum parasites. Anal. Chem. 86, 8814-8821. doi: 10.1021/ac502098w

Che, P., Cui, L., Kutsch, O., Cui, L., and Li, Q. (2012). Validating a firefly luciferasebased high-throughput screening assay for antimalarial drug discovery. Assay Drug Dev. Technol. 10, 61-68. doi: 10.1089/adt.2011.0378

Claser, C., Malleret, B., Gun, S. Y., Wong, A. Y., Chang, Z. W., Teo, P., et al. (2011). $\mathrm{CD} 8+\mathrm{T}$ cells and IFN $-\gamma$ mediate the time-dependent accumulation of infected red blood cells in deep organs during experimental cerebral malaria. PLOS ONE 6:e18720. doi: 10.1371/journal.pone.0018720

Cui, L., Miao, J., Wang, J., Li, Q., and Cui, L. (2008). Plasmodium falciparum: development of a transgenic line for screening antimalarials using firefly luciferase as the reporter. Exp. Parasitol. 120, 80-87. doi: 10.1016/j.exppara.2008. 05.003

de Azevedo, M. F., Gilson, P. R., Gabriel, H. B., Simões, R. F., Angrisano, F., Baum, J., et al. (2012). Systematic analysis of FKBP inducible degradation domain tagging strategies for the human malaria parasite Plasmodium falciparum. PLoS ONE 7:e40981. doi: 10.1371/journal.pone.0040981

Deitsch, K. W., del Pinal, A., and Wellems, T. E. (1999). Intra-cluster recombination and var transcription switches in the antigenic variation of Plasmodium falciparum. Mol. Biochem. Parasitol. 101, 107-116. doi: 10.1016/S01666851(99)00062-6

de Koning-Ward, T. F., Sperança, M. A., Waters, A. P., and Janse, C. J. (1999). Analysis of stage specificity of promoters in Plasmodium berghei using luciferase as a reporter. Mol. Biochem. Parasitol. 100, 141-146. doi: 10.1016/S01666851(99)00042-0

Delves, M. J., and Sinden, R. E. (2010). A semi-automated method for counting fluorescent malaria oocysts increases the throughput of transmission blocking studies. Malar. J. 9, 35. doi: 10.1186/1475-2875-9-35

Derbyshire, E. R., Prudêncio, M., Mota, M. M., and Clardy, J. (2012). Liver-stage malaria parasites vulnerable to diverse chemical scaffolds. Proc. Natl. Acad. Sci. U.S.A. 109, 8511-8516. doi: 10.1073/pnas.1118370109

Ekland, E. H., Schneider, J., and Fidock, D. A. (2011). Identifying apicoplasttargeting antimalarials using high-throughput compatible approaches. FASEB J. 25, 3583-3593. doi: 10.1096/fj.11-187401

Epp, C., Raskolnikov, D., and Deitsch, K. W. (2008). A regulatable transgene expression system for cultured Plasmodium falciparum parasites. Malar. J. 7, 86. doi: $10.1186 / 1475-2875-7-86$

Fidock, D. A. (2010). Drug discovery: priming the antimalarial pipeline. Nature 465, 297-298. doi: 10.1038/465297a
Fernandez-Becerra, C., de Azevedo, M. F., Yamamoto, M. M., and del Portillo, H. A. (2003). Plasmodium falciparum: new vector with bi-directional promoter activity to stably express transgenes. Exp. Parasitol. 103, 88-91. doi: 10.1016/S00144894(03)00065-1

Frank, M., Dzikowski, R., Costantini, D., Amulic, B., Berdougo, E., and Deitsch, K. (2006). Strict pairing of var promoters and introns is required for var gene silencing in the malaria parasite Plasmodium falciparum. J. Biol. Chem. 281, 9942-9952 doi: 10.1074/jbc.M513067200

Franke-Fayard, B., Djokovic, D., Dooren, M. W., Ramesar, J., Waters, A. P., Falade, M. O., et al. (2008). Simple and sensitive antimalarial drug screening in vitro and in vivo using transgenic luciferase expressing Plasmodium berghei parasites. Int. J. Parasitol. 38, 1651-1662. doi: 10.1016/j.ijpara.2008.05.012

Franke-Fayard, B., Janse, C. J., Cunha-Rodrigues, M., Ramesar, J., Büscher, P., Que, I., et al. (2005). Murine malaria parasite sequestration: CD36 is the major receptor, but cerebral pathology is unlinked to sequestration. Proc. Natl. Acad. Sci. U.S.A. 102, 11468-11473. doi: 10.1073/pnas.0503386102

Franke-Fayard, B., Waters, A. P., and Janse, C. J. (2006). Real-time in vivo imaging of transgenic bioluminescent blood stages of rodent malaria parasites in mice. Nat. Protoc. 1, 476-485. doi: 10.1038/nprot.2006.69

Golightly, L. M., Mbacham, W., Daily, J., and Wirth, D. F. (2000). 3' UTR elements enhance expression of Pgs28, an ookinete protein of Plasmodium gallinaceum. Mol. Biochem. Parasitol. 105, 61-70. doi: 10.1016/S0166-6851(99)00165-6

Goonewardene, R., Daily, J., Kaslow, D., Sullivan, T. J., Duffy, P., Carter, R., et al. (1993). Transfection of the malaria parasite and expression of firefly luciferase. Proc. Natl. Acad. Sci. U.S.A. 90, 5234-5236. doi: 10.1073/pnas.90.11.5234

Gopalakrishnan, A. M., and López-Estraño, C. (2010). Role of cis-regulatory elements on the ring-specific hrp3 promoter in the human parasite Plasmodium falciparum. Parasitol Res. 106, 833-845. doi: 10.1007/s00436-010-1738-9

Hasenkamp, S., Russell, K. T., and Horrocks, P. (2012). Comparison of the absolute and relative efficiencies of electroporation-based transfection protocols for Plasmodium falciparum. Malar. J. 11, 210. doi: 10.1186/1475-2875-11-210

Hasenkamp, S., Russell, K. T., Ullah, I., and Horrocks, P. (2013a). Functional analysis of the 5' untranslated region of the phosphoglutamase 2 transcript in Plasmodium falciparum. Acta Trop. 127, 69-74. doi: 10.1016/j.actatropica.2013.03.007

Hasenkamp, S., Sidaway, A., Devine, O., Roye, R., and Horrocks, P. (2013b). Evaluation of bioluminescence-based assays of anti-malarial drug activity. Malar. J. 12, 58. doi: 10.1186/1475-2875-12-58

Helm, S., Lehmann, C., Nagel, A., Stanway, R. R., Horstmann, S., Llinas, M., et al. (2010). Identification and characterization of a liver stage-specific promoter region of the malaria parasite Plasmodium. PLoS ONE 5:e13653. doi: 10.1371/journal.pone.0013653

Horrocks, P., and Kilbey, B. J. (1996). Physical and functional mapping of the transcriptional start sites of Plasmodium falciparum proliferating cell nuclear antigen. Mol. Biochem. Parasitol. 82, 207-215. doi: 10.1016/0166-6851(96)02737-5

Imai, T., Iwawaki, T., Akai, R., Suzue, K., Hirai, M., Taniguchi, T., et al. (2014). Evaluating experimental cerebral malaria using oxidative stress indicator OKD48 mice. Int. J. Parasitol. 44, 681-685. doi: 10.1016/j.ijpara.2014.06.002

Kaushansky, A., Mikolajczak, S. A., Vignali, M., and Kappe, S. H. (2014). Of men in mice: the success and promise of humanized mouse models for human malaria parasite infections. Cell Microbiol. 16, 602-611. doi: 10.1111/cmi.12277

Khan, T., van Brummelen, A. C., Parkinson, C. J., and Hoppe, H. C. (2012). ATP and luciferase assays to determine the rate of drug action in in vitro cultures of Plasmodium falciparum. Malar. J. 11, 369. doi: 10.1186/1475-2875-11-369

Kolevzon, N., Nasereddin, A., Naik, S., Yavin, E., and Dzikowski, R. (2014). Use of peptide nucleic acids to manipulate gene expression in the malaria parasite Plasmodium falciparum. PLoS ONE 9:e86802. doi: 10.1371/journal.pone. 0086802

Lacrue, A. N., Sáenz, F. E., Cross, R. M., Udenze, K. O., Monastyrskyi, A., Stein, S., et al. (2013). 4(1H)-Quinolones with liver stage activity against Plasmodium berghei. Antimicrob. Agents. Chemother. 57, 417-424. doi: 10.1128/AAC. 00793-12

Li, Q., O’Neil, M., Xie, L., Caridha, D., Zeng, Q., Zhang, J., et al. (2014). Assessment of the prophylactic activity and pharmacokinetic profile of oral tafenoquine compared to primaquine for inhibition of liver stage malaria infections. Malar. J. 13, 141. doi: 10.1186/1475-2875-13141

Lin, J. W., Sajid, M., Ramesar, J., Khan, S. M., Janse, C. J., and Franke-Fayard, B. (2013). Screening inhibitors of $P$. berghei blood stages using bioluminescent 
reporter parasites. Methods Mol. Biol. 923, 507-522. doi: 10.1007/978-1-62703026-7_35

López-Estraño, C., Gopalakrishnan, A. M., Semblat, J. P., Fergus, M. R., Mazier, D., and Haldar, K. (2007). An enhancer-like region regulates hrp3 promoter stage-specific gene expression in the human malaria parasite Plasmodium falciparum. Biochim. Biophys. Acta 1769, 506-513. doi: 10.1016/j.bbaexp.2007. 04.009

Lucantoni, L., Duffy, S., Adjalley, S. H., Fidock, D. A., and Avery, V. M. (2013). Identification of MMV malaria box inhibitors of Plasmodium falciparum earlystage gametocytes using a luciferase-based high-throughput assay. Antimicrob. Agents Chemother. 57, 6050-6062. doi: 10.1128/AAC.00870-13

Lucumi, E., Darling, C., Jo, H., Napper, A. D., Chandramohanadas, R., Fisher, N., et al. (2010). Discovery of potent small-molecule inhibitors of multidrugresistant Plasmodium falciparum using a novel miniaturized high-throughput luciferase-based assay. Antimicrob. Agents Chemother. 54, 3597-3604. doi: 10.1128/AAC.00431-10

Mair, G. R., Braks, J. A., Garver, L. S., Wiegant, J. C., Hall, N., Dirks, R. W., et al. (2006). Regulation of sexual development of Plasmodium by translational repression. Science 313, 667-669 doi: 10.1126/science.1125129

Manzoni, G., Briquet, S., Risco-Castillo, V., Gaultier, C., Topçu, S., Ivãnescu, M. L., et al. (2014). A rapid and robust selection procedure for generating drugselectable marker-free recombinant malaria parasites. Sci. Rep. 4, 4760. doi: 10.1038/srep04760

Marcsisin, S. R., Sousa, J. C., Reichard, G. A., Caridha, D., Zeng, Q., Roncal, N., et al. (2014). Tafenoquine and NPC-1161B require CYP 2D metabolism for antimalarial activity: implications for the 8-aminoquinoline class of anti-malarial compounds. Malar. J. 13, 2. doi: 10.1186/1475-2875-13-2

Militello, K. T., Dodge, M., Bethke, L., and Wirth, D. F. (2004). Identification of regulatory elements in the Plasmodium falciparum genome. Mol. Biochem. Parasitol. 134, 75-88. doi: 10.1016/j.molbiopara.2003.11.004

Militello, K. T., and Wirth, D. F. (2003). A new reporter gene for transient transfection of Plasmodium falciparum. Parasitol. Res. 89, 154-157. doi: 10.1007/s00436-002-0721-5

Miller, J. L., Murray, S., Vaughan, A. M., Harupa, A., Sack, B., Baldwin, M., et al. (2013). Quantitative bioluminescent imaging of pre-erythrocytic malaria parasite infection using luciferase-expressing Plasmodium yoelii. PLoS ONE 8:e60820. doi: 10.1371/journal.pone.0060820

Miller, L. H., Baruch, D. I., Marsh, K., and Doumbo, O. K. (2002). The pathogenic basis of malaria. Nature 415, 673-679. doi: 10.1038/415673a

Milner, D. A. Jr., Whitten, R. O., Kamiza, S., Carr, R., Liomba, G., Dzamalala, C., et al. (2014). The systemic pathology of cerebral malaria in African children. Front. Cell Infect Microbiol. 4:104. doi: 10.3389/fcimb.2014.00104

Myrick, A., Munasinghe, A., Patankar, S., and Wirth, D. F. (2003). Mapping of the Plasmodium falciparum multidrug resistance gene 5'-upstream region, and evidence of induction of transcript levels by antimalarial drugs in chloroquine sensitive parasites. Mol. Microbiol. 49, 671-683. doi: 10.1046/j.13652958.2003.03597.x

Muhle, R. A., Adjalley, S., Falkard, B., Nkrumah, L. J., Muhle, M. E., and Fidock, D. A. (2009). A var gene promoter implicated in severe malaria nucleates silencing and is regulated by 3' untranslated region and intronic cis-elements. Int. J. Parasitol. 39, 1425-1439. doi: 10.1016/j.ijpara.2009.05.001

Mwakingwe, A., Ting, L. M., Hochman, S., Chen, J., Sinnis, P., and Kim, K. (2009). Non-invasive real-time monitoring of liver-stage development of bioluminescent Plasmodium parasites. J. Infect. Dis. 200, 1470-1478. doi: 10.1086/ 606115

Oguariri, R. M., Dunn, J. M., and Golightly, L. M. (2006). 3' gene regulatory elements required for expression of the Plasmodium falciparum developmental protein, Pfs25. Mol. Biochem. Parasitol. 146, 163-172. doi: 10.1016/j.molbiopara.2005.12.004

Ozwara, H., van der Wel, A., Kocken, C. H., and Thomas, A. W. (2003). Heterologous promoter activity in stable and transient Plasmodium knowlesi transgenes. Mol. Biochem. Parasitol. 130, 61-64. doi: 10.1016/S0166-6851(03)00141-5

Pasini, E. M., Braks, J. A., Fonager, J., Klop, O., Aime, E., Spaccapelo, R., et al. (2013). Proteomic and genetic analyses demonstrate that Plasmodium berghei blood stages export a large and diverse repertoire of proteins. Mol. Cell. Proteomics 12, 426-448. doi: 10.1074/mcp.M112. 021238
Patakottu, B. R., Singh, P. K., Malhotra, P., Chauhan, V. S., and Patankar, S. (2012). In vivo analysis of translation initiation sites in Plasmodium falciparum. Mol. Biol. Rep. 39, 2225-2232. doi: 10.1007/s11033-011-0971-3

Ploemen, I., Behet, M., Nganou-Makamdop, K., van Gemert, G. J., Bijker, E., Hermsen, C., et al. (2011). Evaluation of immunity against malaria using luciferase-expressing Plasmodium berghei parasites. Malar. J. 10, 350. doi: 10.1186/1475-2875-10-350

Ploemen, I. H., Chakravarty, S., van Gemert, G. J., Annoura, T., Khan, S. M., Janse, C. J., et al. (2013). Plasmodium liver load following parenteral sporozoite administration in rodents. Vaccine. 31, 3410-3416. doi: 10.1016/j.vaccine.2012.09.080

Ploemen, I. H., Prudêncio, M., Douradinha, B. G., Ramesar, J., Fonager, J., van Gemert, G. J., et al. (2009). Visualisation and quantitative analysis of the rodent malaria liver stage by real time imaging. PLOS ONE 4:e7881. doi: 10.1371/journal.pone.0007881

Porter, M. E. (2002). Positive and negative effects of deletions and mutations within the 5' flanking sequences of Plasmodium falciparum DNA polymerase delta. Mol. Biochem. Parasitol. 122, 9-19. doi: 10.1016/S0166-6851(02)00064-6

Ramakrishnan, C., Rademacher, A., Soichot, J., Costa, G., Waters, A. P., Janse, C. J., et al. (2012). Salivary gland-specific P. berghei reporter lines enable rapid evaluation of tissue-specific sporozoite loads in mosquitoes. PLoS ONE. 7:e36376. doi: 10.1371/journal.pone.0036376

Ramalhete, C., da Cruz, F. P., Lopes, D., Mulhovo, S., Rosário, V. E., Prudêncio, M., et al. (2011). Triterpenoids as inhibitors of erythrocytic and liver stages of Plasmodium infections. Bioorg. Med. Chem. 19, 7474-7481. doi: 10.1016/j.bmc.2011.10.044.

Ramalhete, C., da Cruz, F. P., Mulhovo, S., Sousa, I. J., Fernandes, M. X., Prudêncio, M., et al. (2014). Dual-stage triterpenoids from an African medicinal plant targeting the malaria parasite. Bioorg. Med. Chem. 22, 3887-3890. doi: 10.1016/j.bmc.2014.06.019

Sack, B. K., Miller, J. L., Vaughan, A. M., Douglass, A., Kaushansky, A., Mikolajczak, S., et al. (2014). Model for in vivo assessment of humoral protection against malaria sporozoite challenge by passive transfer of monoclonal antibodies and immune serum. Infect. Immun. 82, 808-817. doi: 10.1128/IAI.01249-13

Smale, S. T. (2010). Luciferase assay. Cold Spring Harb. Protoc. 2010. doi: $10.1101 /$ pdb.prot5421

Spaccapelo, R., Janse, C. J., Caterbi, S., Franke-Fayard, B., Bonilla, J. A., Syphard, L. M., et al. (2010). Plasmepsin 4-deficient Plasmodium berghei are virulence attenuated and induce protective immunity against experimental malaria. Am. J. Pathol. 176, 205-217. doi: 10.2353/ajpath.2010.090504

Stone, W. J., Churcher, T. S., Graumans, W., van Gemert, G. J., Vos, M. W., Lanke, K. H., et al. (2014). A scalable assessment of Plasmodium falciparum transmission in the standard membrane-feeding assay, using transgenic parasites expressing green fluorescent protein-luciferase. J. Infect. Dis. 210, 1456-1463. doi: 10.1093/infdis/jiu271

Thorne, N., Shen, M., Lea, W. A., Simeonov, A., Lovell, S., Auld, D. S., et al. (2012). Firefly luciferase in chemical biology: a compendium of inhibitors, mechanistic evaluation of chemotypes, and suggested use as a reporter. Chem. Biol. 19, 1060-1072. doi: 10.1016/j.chembiol.2012.07.015

Van Reet, N., Van de Vyver, H., Pyana, P. P., Van der Linden, A. M., and Büscher, P. (2014). A panel of Trypanosoma brucei strains tagged with blue and red-shifted luciferases for bioluminescent imaging in murine infection models. PLoS Negl. Trop. Dis. 8:e3054. doi: 10.1371/journal.pntd.0003054

van Schaijk, B. C., Ploemen, I. H., Annoura, T., Vos, M. W., Lander, F., van Gemert, G. J., et al. (2014). A genetically attenuated malaria vaccine candidate based on P. falciparum $69 /$ slarp gene-deficient sporozoites. Elife 19:3. doi: 10.7554/eLife.03582

Vaughan, A. M., Mikolajczak, S. A., Camargo, N., Lakshmanan, V., Kennedy, M., Lindner, S. E., et al. (2012). A transgenic Plasmodium falciparum NF54 strain that expresses GFP-luciferase throughout the parasite life cycle. Mol. Biochem. Parasitol. 186, 143-147. doi: 10.1016/j.molbiopara.2012. 10.004

Waller, K. L., Muhle, R. A., Ursos, L. M., Horrocks, P., Verdier-Pinard, D., Sidhu, A. B., et al. (2003). Chloroquine resistance modulated in vitro by expression levels of the Plasmodium falciparum chloroquine resistance transporter. J. Biol. Chem. 278, 33593-33601. doi: 10.1074/jbc. M302215200 
Waters, A. P., Thomas, A. W., van Dijk, M. R., and Janse, C. J. (1997). Transfection of malaria parasites. Methods 13, 134-147. doi: 10.1006/meth.1997.0506

Weiwer, M., Mulrooney, C., Massi, D., Heidebrecht, R., Wiegand, R., Lukens, A. K., et al. (2011). ML238: An Antimalarial Small Molecule of a Unique Structural Class. Probe Reports. Bethesda, MD: NIH Molecular Libraries Program.

White, N. J., Pukrittayakamee, S., Hien, T. T., Faiz, M. A., Mokuolu, O. A., and Dondorp, A. M. (2014). Malaria. Lancet 383, 723-735. doi: 10.1016/S01406736(13)60024-0

World Health Organization. (2013). World Malaria Report. Available at: http:// www.who.int/malaria/publications/worldmalariareport/en/

Zhang, X., Tolzmann, C. A., Melcher, M., Haas, B. J., Gardner, M. J., Smith, J. D., et al. (2011). Branch point identification and sequence requirements for intron splicing in Plasmodium falciparum. Eukaryot Cell. 10, 1422-1428. doi: 10.1128/EC.05193-11
Zuzarte-Luis, V., Sales-Dias, J., and Mota, M. M. (2014). Simple, sensitive and quantitative bioluminescence assay for determination of malaria pre-patent period. Malar. J. 13, 15. doi: 10.1186/1475-2875-13-15

Conflict of Interest Statement: The authors declare that the research was conducted in the absence of any commercial or financial relationships that could be construed as a potential conflict of interest.

Copyright $\odot 2015$ Siciliano and Alano. This is an open-access article distributed under the terms of the Creative Commons Attribution License (CC BY). The use, distribution or reproduction in other forums is permitted, provided the original author(s) or licensor are credited and that the original publication in this journal is cited, in accordance with accepted academic practice. No use, distribution or reproduction is permitted which does not comply with these terms. 